\title{
Networks on the edge of chaos: Global feedback control of turbulence in oscillator networks
}

\author{
Santiago Gil* and Alexander S. Mikhailov \\ Abteilung Physikalishe Chemie, Fritz-Haber-Institut der Max-Planck-Gesellschaft, Faradayweg 4-6, 14195 Berlin, Germany \\ (Received 12 August 2008; revised manuscript received 28 November 2008; published 25 February 2009)
}

\begin{abstract}
Random networks of coupled phase oscillators with phase shifts in the interaction functions are considered. In such systems, extensive chaos (turbulence) is observed in a wide range of parameters. We show that, by introducing global feedback, the turbulence can be suppressed and a transition to synchronous oscillations can be induced. Our attention is focused on the transition scenario and the properties of patterns, including intermittent turbulence, which are found at the edge of chaos. The emerging coherent patterns represent various self-organized active (sub)networks whose size and behavior can be controlled.
\end{abstract}

DOI: 10.1103/PhysRevE.79.026219

PACS number(s): 05.45.Xt, 89.75.Fb, 05.45.Gg, 89.75.Hc

\section{INTRODUCTION}

Network-organized nonlinear systems are ubiquitous in nature and in the human world [1]. They may well be even more important with respect to applications than continuous nonlinear media, such as reaction-diffusion systems, which have been extensively studied. Understanding principal modes of self-organization in network-organized systems and finding ways of controlling them is a major scientific challenge. While studies on network dynamics are rapidly progressing (see Refs. [2,3]), only some of its aspects have been so far mainly considered. Studies are often focused on linear stability of synchronous oscillations in such systems and its dependence on the network architecture. Comparing this to studies of nonequilibrium pattern formation in nonlinear continuous reaction-diffusion models [4-7], one can notice that other kinds of coherent dynamical patterns, beyond of uniform oscillations, are possible too. What would be the analogs of traveling or standing waves, Turing patterns, or high-dimensional chaos (turbulence) for the networkorganized dynamical systems? What are the bifurcations corresponding to transitions between different coherent patterns in the networks? How would the emergence and properties of self-organized coherent patterns or turbulence in dynamical network systems be controlled? Many of such questions are awaiting their analysis.

Our study is motivated by previous investigations on continuous nonequilibrium media. Diffusive coupling between periodic oscillators on a lattice or in a continuous medium can destabilize uniform oscillations and lead to diffusioninduced extensive chaos, known as chemical turbulence $[5,8]$. In experimental and theoretical investigations [9-13], it has been shown that this kind of turbulence can be controlled by introducing global feedback. Such feedback can not only induce uniform oscillations, but also produce various coherent dynamical patterns, observed at the edge of chaos within the transition region.

Here we consider the network analog of the problem studied in Ref. [11]. We take a network system where individual elements, occupying the nodes of a network, are periodic phase oscillators. We introduce interactions between the os-

\footnotetext{
*gil@fhi-berlin.mpg.de
}

cillators, which depend only on the differences in the phase states of the network neighbors and thus correspond to the diffusive coupling in continuous media. We show, by direct computation of Lyapunov exponents and embedding dimensions, that such interactions are sufficient to break down uniform oscillations and to establish high-dimensional chaos (network turbulence). We introduce global feedback and demonstrate that, through its action, not only synchronization can be restored, but also various, complexly organized dynamical regimes can be achieved. Then, we systematically study coherent structures and intermittent turbulence in the transition from developed network turbulence to synchronous oscillations under the variation of the global feedback intensity.

Our analysis reveals that the transition can be divided into two distinct stages. In the first stage, groups of elements become active, that they repeatedly perform excursions from the common synchronous state. These groups are organized into (sub)networks whose collective dynamics depends on the size of a (sub)network and its architecture. As the feedback intensity is decreased, the fraction of active elements grows. The networks formed by these elements increase in size and become more numerous. When a certain feedback intensity is reached, such networks merge in a percolation transition, so that a large connected network of active elements is formed. Even before this transition, network dynamics becomes chaotic, as signalled by positive values of the maximal Lyapunov exponent.

In the next stage, changes in the internal organization of the dynamical system are best monitored by looking at the global Kuramoto order parameter which specifies the observed degree of synchronization. We find that, as the feedback intensity is further decreased, this order parameter rapidly decreases and becomes very small below a certain finite threshold. Because this global parameter determines the magnitude of the global feedback signal in the system, its vanishing indicates the failure of the global feedback to entrain the system. Our calculations show that the maximal Lyapunov exponents and the Kaplan-Yorke embedding dimension of the system strongly increase as the feedback breakdown transition is approached.

After this transition has taken place, all system elements still receive relatively strong signals from their network neighbors and they do not become effectively independent. Analyzing the internal organization of the system in this tur- 
bulent state, we find that it is characterized by repeated synchronization bursts that involve only small groups of network elements. Remarkably, each next burst appears to be produced by a different group of network elements.

The considered model is formulated in the next section. The chaos transition scenario is outlined in Sec. III. This is followed by a more detailed numerical analysis of the system behavior at two characteristic stages in the subsequent two sections. The paper ends with conclusions and discussion of obtained results.

\section{THE MODEL}

We consider a dynamical system representing a network of $N$ identical phase oscillators with phase-shifted pairwise interactions. It is described by a set of differential equations

$$
\dot{\phi}_{i}=\omega+\frac{1}{\rho N} \sum_{j=1, j \neq i}^{N} T_{i j} \sin \left(\phi_{j}-\phi_{i}+2 \pi A_{i j}\right)
$$

Because all oscillators are identical, the common natural frequency can be assumed equal to zero $(\omega=0)$, which corresponds to the use of a corotating reference frame. $T$ is the adjacency matrix of the interaction network and $\rho$ is its average connectivity $\left\langle T_{i, j}\right\rangle$, so that $\rho N$ is the mean degree of a node. The adjacency matrix is not symmetric and therefore directed interactions are present in the system. Interactions between the elements are characterized by random phase shifts $2 \pi A_{i j}$. Since directed interactions are considered, the phase shifts are generally different in two directions $i \rightarrow j$ and $j \rightarrow i$. Note that in the globally coupled case $\left(T_{i j}=1\right.$ for any $i$ and $j$ ) and for phase shifts taking only one of two values $A_{i j}=0$ or $A_{i j}=1 / 2$, the considered model becomes reduced to that previously studied by Daido [14]. However, in contrast to the Daido model, our system is not variational and therefore its dynamical properties are significantly different. Note also that the model (1) can be derived in the weak interaction limit from the model of coupled phase oscillators with time delays (see Ref. [15]); however, we do not perform such a reduction in this paper and take Eq. (1) as the starting point of our analysis.

The matrix of phase shifts is chosen as $A_{i j}=a_{i j} \delta$, where $a_{i j}$ are random numbers independently drawn from the interval $[0,1]$ for any pair $(i, j)$. The parameter $\delta$ specifies the characteristic magnitude of the phase shifts in the model. Our numerical investigations show that, for sufficiently large values of $\delta$, network dynamics is chaotic and has a large embedding dimension, so that high-dimensional chaos (turbulence) is observed in the system. In the numerical simulations that follow, the value $\delta=0.4$ is chosen, a value high enough to induce chaotic dynamics.

To control the behavior of the network, global feedback is used in our investigations. Generally, global feedback control means that information about the states of all elements in a system is gathered and used to generate a common (global) control signal which is applied back to all elements [13]. For our study, the global signal shall be chosen as

$$
Z=\frac{1}{N} \sum_{i=1}^{N} \exp \left(\iota \phi_{i}\right)
$$

Thus, it coincides with the Kuramoto order parameter [8] used to measure the degree of synchronization in a system of oscillators. We assume that the global signal is applied as an external force to all oscillators in the network

$$
\dot{\phi}_{i}=\frac{1}{\rho N} \sum_{j=1, j \neq i}^{N} T_{i j} \sin \left(\phi_{j}-\phi_{i}+2 \pi A_{i j}\right)+\frac{\mu}{2 i}\left(Z e^{-i \phi_{i}}-\text { c.c. }\right),
$$

where $\mu$ is the parameter that specifies feedback intensity.

Introducing the magnitude $R$ and the phase $\Phi$ of the global signal as $Z=R \exp (i \Phi)$, we arrive at the final set of equations that describe the considered model

$$
\dot{\phi}_{i}=\frac{1}{\rho N} \sum_{j=1, j \neq i}^{N} T_{i j} \sin \left(\phi_{j}-\phi_{i}+2 \pi A_{i j}\right)+\mu R \sin \left(\Phi-\phi_{i}\right) .
$$

In our investigations, we will perform numerical simulations of systems composed of $N=1000$ identical oscillators. As a pattern of interactions $T$, standard networks of the ErdösRenyi type will be used. In such networks, (directed) links connecting two elements $(i \rightarrow j)$ are chosen independently at random with some probability $\rho$. Explicitly, the elements of matrix $T$ are

$$
T_{i j}=\left\{\begin{array}{l}
1 \quad \text { with probability } \rho, \\
0 \quad \text { with probability } 1-\rho,
\end{array}\right.
$$

where the first condition excludes loop edges. Therefore, in the limit of large networks the mean degree of a node is $\langle k\rangle=\rho N$. For our simulations, we have set $\rho=0.006$, having checked that, despite the low connectivity, the considered networks are still fully connected, so that each node can be reached from any other node in the network.

\section{LYAPUNOV EXPONENTS AND KAPLAN-YORKE DIMENSION}

To characterize chaotic dynamics, two measures have been chosen in our investigations. The first of them is the maximal Lyapunov exponent that measures the rate of the exponential divergence of the trajectories. The second is the Kaplan-Yorke embedding dimension, which tells what is the minimal number of independent variables generally needed to describe the chaotic attractor [16].

Lyapunov exponents are a set of numbers $\lambda_{i}$ that characterize the average rate of local divergence (or convergence) of close trajectories within an attractor. In particular, the maximal Lyapunov exponent $\lambda_{1}$ is the largest of these numbers and it indicates whether neighboring trajectories separate or converge in time. For the calculation of the full spectrum of Lyapunov exponents, we shall follow the method developed by Benettin et al. [17] and Shimada and Nagashima [18] (see also Ref. [19]).

The maximal Lyapunov exponent can be computed by tracking the evolution of small perturbations to an orbit be- 


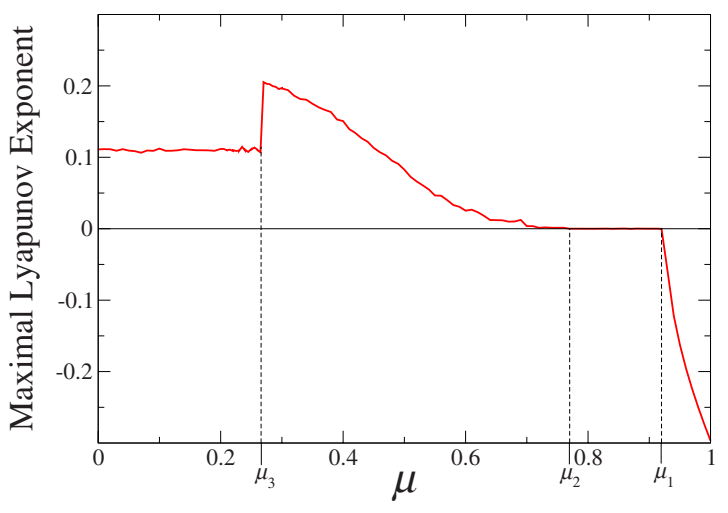

FIG. 1. (Color online) Dependence of the maximal Lyapunov exponent on the control parameter $\mu$.

longing to the attractor. For this, it is convenient to use the linearized form of the evolution equations. This amounts to observing the evolution of a perturbation vector $\vec{q}_{1}$ that obeys

$$
\frac{\partial}{\partial t} \vec{q}_{1}(t)=J\left(\phi_{i}(t)\right) \vec{q}_{1}(t)
$$

where $J$ is the Jacobian matrix of the system. Independently of the initial condition $\vec{q}_{1}(0)$, this vector tends to adopt the direction of maximal elongation (or minimal compression) in phase space. The average rate of growth of the module of $\vec{q}_{1}(t)$ over the attractor yields the maximal Lyapunov exponent.

The remaining Lyapunov exponents can be calculated by restricting the possible directions of perturbations. For example, $\lambda_{2}$ measures the rate of growth of a perturbation $\vec{q}_{2}$ in a direction orthogonal to $\vec{q}_{1}$. Generally, $\lambda_{i}$ represents the average growth rate of a perturbation vector $\vec{q}_{i}$ which belongs to the subspace orthogonal to vectors $\vec{q}_{1}, \ldots, \vec{q}_{i-1}$. It is clear then that the Lyapunov exponents calculated in this way satisfy the condition $\lambda_{1}>\lambda_{2}>\cdots>\lambda_{N-1}>\lambda_{N}$.

In numerical implementations, several precautions need to be taken [20]. In our case, in particular, we know that the system is invariant under rigid translations, that is, under transformations of the variables $\phi_{i}^{\prime} \rightarrow \phi_{i}+C$ for all $i$. This means that perturbations along the direction $\vec{q}_{1}=(1, \ldots, 1)$ will remain unchanged, resulting in an exponent equal to 0 . These perturbations provide no information on the dynamics of the system, and we therefore force $\vec{q}_{1}$ to be orthogonal to the vector $(1, \ldots, 1)$ in every time step, letting it evolve freely in all other directions. This means that the Lyapunov spectrum of our system will be defined by $N-1$ exponents, excluding the zero exponent that is always present.

In this way, the entire spectrum of Lyapunov exponents can be calculated. An important quantity to derive from the spectrum is the dimensionality of the attractor. This is calculated using the Kaplan-Yorke definition [21] which reads

$$
D=k+\frac{\sum_{i=1}^{k} \lambda_{i}}{\left|\lambda_{k+1}\right|}
$$

where $k$ is such that $\sum_{i=1}^{k} \lambda_{i} \geq 0$ but $\sum_{i=1}^{k+1} \lambda_{i}<0$. The dimension is in general a noninteger number. This means that the small-

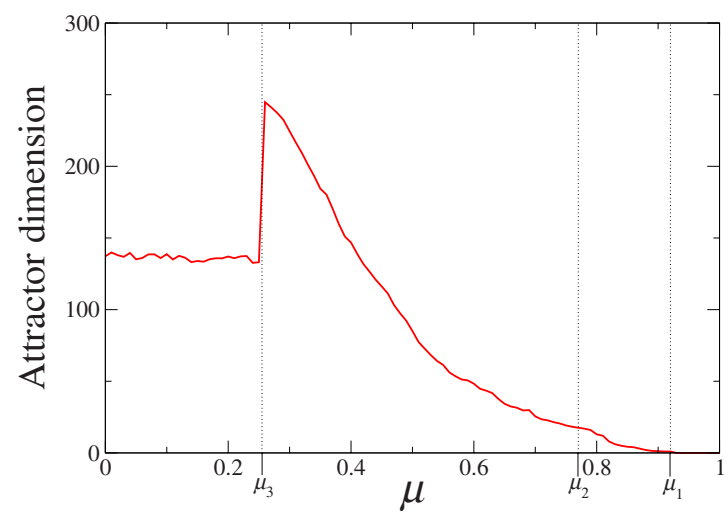

FIG. 2. (Color online) Dependence of the Kaplan-Yorke embedding dimension on the control parameter $\mu$.

est integer larger than or equal to $D$ is the number of independent variables that would be necessary to describe the dynamics within the attractor.

The maximal Lyapunov exponent and the Kaplan-Yorke dimension are shown as functions of the control parameter $\mu$ in Figs. 1 and 2, respectively. Additionally, four histograms of the distributions of all $N-1$ Lyapunov exponents are shown in Fig. 3 for different values of $\mu$. In Fig. 1, we can immediately see that the maximal Lyapunov exponent is positive in the absence of global feedback, that is, at $\mu=0$. This implies that the behavior is chaotic, as anticipated in the previous section. Furthermore, the embedding dimension of this chaotic attractor is high $(D=137.2$ at $\mu=0)$ and comparable to the dimension (size) of the system $N=1000$. Thus, our system can be considered as exhibiting network turbulence in absence of the feedback.

In contrast, for large values of $\mu$ we can see that $\lambda_{1}$ is negative, indicating the presence of a stable fixed-point attractor. The dimension is accordingly $D=0$. This corresponds to a state of synchronization, induced by the global

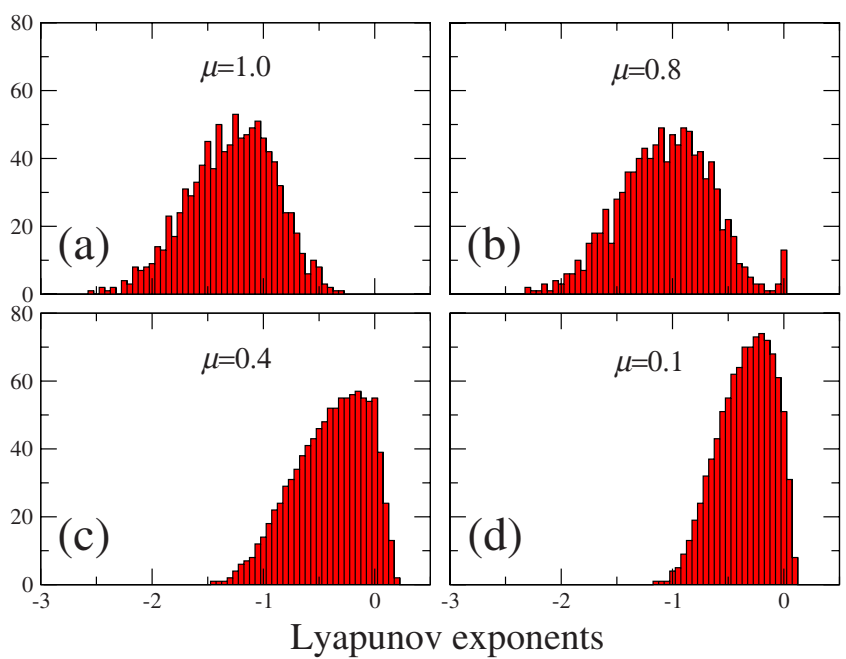

FIG. 3. (Color online) Histograms of Lyapunov exponents for different values of the control parameter $\mu$. 
feedback. ${ }^{1}$ The distribution of Lyapunov exponents at $\mu=1.0$ is approximately symmetric and roughly centered at -1.3 [Fig. 3(a)].

When the intensity of the global feedback is decreased, the synchronous stationary state becomes unstable at $\mu_{1}$ $\approx 0.92$ and we get $\lambda_{1}=0$ (Fig. 1). The maximal Lyapunov exponent remains equal to zero in an interval of $\mu$ below this transition, so that limit-cycle oscillations take place there. It is interesting that the Kaplan-Yorke dimension of the attractor grows below $\mu=\mu_{1}$, even though chaos is absent. This indicates that the system has several zero Lyapunov exponents, including $\lambda_{1}$ [indeed, if $k$ zero Lyapunov exponents are present, $D=k$ according to Eq. (7)]. The presence of degenerate zero Lyapunov exponents is directly demonstrated by Fig. 3(b), where a clear peak is present at 0 .

The attractor becomes chaotic at $\mu_{2} \approx 0.77$, where $\lambda_{1}$ first becomes positive. Both the value of the maximal Lyapunov exponent and the dimension of the attractor grow monotonously when decreasing the intensity of the global feedback. Thus, the behavior becomes more complex as chaos develops. The dimension reaches high values, up to $D=244.8$, significantly higher than that in the absence of global feedback. As seen in Fig. 3(c), a large number of Lyapunov exponents are then positive (approximately 10\% of them) and the distribution is now clearly asymmetric.

An abrupt drop occurs in both the maximal Lyapunov exponent and the Kaplan-Yorke dimension at about $\mu_{3}=0.26$, indicating a drastic change in the properties of the attractor. The distribution of Lyapunov exponents becomes more narrow and the fraction of positive exponents is approximately $6 \%$. Remarkably, further changes in $\mu$ below this critical value seem to have no effect on the behavior of the system. This suggests that the sudden drop is associated with the breakdown of the global feedback.

The results displayed in these figures indicate that there are several interesting aspects of the transition from synchronization to chaos. In the following we study this transition using different statistical tools to better describe and understand the complex behavior observed in networks at the edge of chaos.

\section{VELOCITY DISTRIBUTIONS AND ACTIVE ELEMENTS}

The global feedback in the considered system tends to impose synchronization. If only global interactions corresponding to this feedback are present, the system undergoes complete synchronization, with all phases becoming identical, $\phi_{i}=\Phi=$ const. In the presence of network interactions, this state is no longer possible because of the phase shifts in the interactions between individual oscillators, which bring the system to a state of frustration [14]. Instead, a state of frequency synchronization can be reached where $\dot{\phi}=\Omega=$ const. If this state is stable, all deviations from it are

\footnotetext{
${ }^{1}$ The synchronous state is invariant with respect to uniform shifts of all phases. This is reflected in the presence of the additional trivial zero Lyapnov exponent, which is discarded.
}

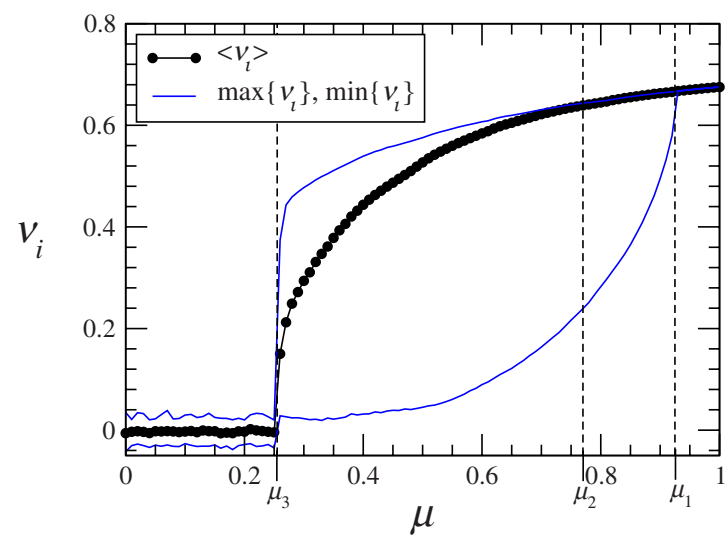

FIG. 4. (Color online) Mean time-averaged velocity of the network (dots) as function of the feedback intensity $\mu$. Thin lines show the maximum and minimum velocity values.

damped, except for a perturbation representing a uniform shift of all phases. Thus, it will be characterized by negative Lyapunov exponents (the trivial zero Lyapunov exponent corresponding to uniform phase shifts is discarded).

As follows from Figs. 1 and 3(a), frequency synchronization takes place in the considered system if global feedback is strong enough, i.e., if $\mu>\mu_{1}$. For weaker feedbacks, this state is destroyed and internal dynamics develops in the system. To monitor the gradual destruction of frequency synchronization, it is natural to observe the behavior of the velocities $\dot{\phi}_{i}$ of network elements. Below we consider longtime average velocities $\nu_{i}$, defined as

$$
\nu_{i}=\frac{1}{\Delta T} \int_{0}^{\Delta T} \dot{\phi}_{i}(t) d t=\frac{1}{\Delta T}\left[\phi_{i}(\Delta T)-\phi_{i}(0)\right],
$$

where the time interval $\Delta T$ is large.

The mean, maximum and minimum values of all velocities $\nu_{i}$ at different feedback intensities $\mu$ have been computed (Fig. 4). We see that for $\mu>\mu_{1}$ the maximum and minimum velocities coincide, so that all velocities are equal and the system is in the state of velocity synchronization. At $\mu<\mu_{3}$, the mean velocity is close to zero $\left\langle\nu_{i}\right\rangle \approx 0$ and dispersion of velocities is small. Inside the transition region $\mu_{1}>\mu>\mu_{3}$, the velocities of individual oscillators are spread broadly.

To characterize the behavior of the system in the transition region, distributions of velocities at three different values of the feedback intensity $\mu$ have been determined (Fig. 5 ). We see that, while the overall spread in the velocities is large, the majority of the oscillators possess the same longterm average velocity, and only a small fraction of them have velocities that are different.

In the reference frame that rotates with the velocity of the distribution peak, one would see that most of the oscillators remain still or at most vibrate around a fixed point, so that their time-averaged velocities are all zero. Therefore, these elements can be described as forming a synchronous condensate. In contrast, the oscillators outside of this group perform repeated phase rotations around the condensate and can be described as active elements. 


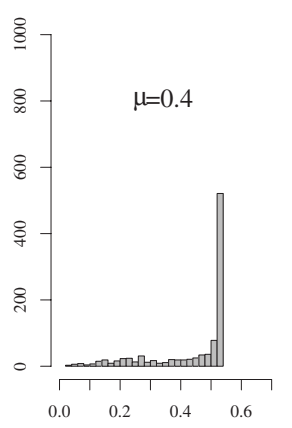

(a)

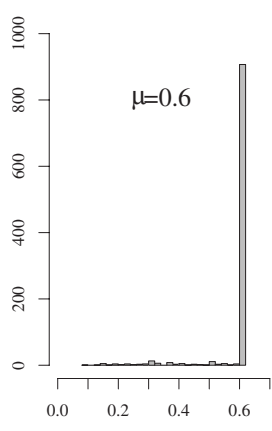

(b)

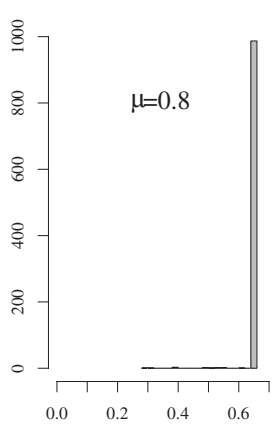

(c)

$$
\dot{\psi}_{i}=-\Omega+\frac{1}{2 i}\left[\left(\widetilde{z}_{i}+\mu R\right) e^{-i \psi_{i}}-\text { c.c. }\right]
$$

where

$$
\widetilde{z_{i}}=\frac{1}{\rho N} \sum_{j=1, j \neq i}^{N} T_{i j} e^{i\left(\psi_{j}+2 \pi A_{i j}\right)}
$$

and $\Omega=\Phi$ is the global velocity. Finally, we can write them in the form

$$
\dot{\psi}_{i}=-\Omega+\mu R_{i} \sin \left(\Psi_{i}-\psi_{i}\right)
$$

with $R_{i} \exp \left(i \Psi_{i}\right)=R+\mu^{-1} \widetilde{z}_{i}$.

In the synchronous state $\psi_{i}=0$ and, therefore, relative phases of all oscillators are determined by a set of algebraic equations

$$
\sin \left(\Psi_{i}-\psi_{i}\right)=\frac{\Omega}{\mu R_{i}} .
$$

A solution to these equations exists only if $\mu R_{i} \geqslant \Omega$ for all nodes $i$. This is always the case if the global feedback is strong $(\mu \rightarrow \infty)$ and contributions from the local signals are negligible. As the global feedback gets weaker, the ratio $\Omega / \mu R_{i}$ increases and, eventually, becomes larger than unity for some of the oscillators. These oscillators can no longer be entrained by the condensate and start to orbit around it, generating phase slips. They form the subset of active elements. When orbiting elements have appeared, they act back on the elements in the condensate and force them to undergo small temporal deviations from the synchronous state. This behavior can be seen in Fig. 6 .

The above analogy with spiking neurons is not far fetched. The transition to orbiting, described by Eq. (13), represents the saddle-node bifurcation on the invariant cycle which is characteristic to type-I neuron models [22,23]. Entrained elements are effectively excitable and can make a rotation (i.e., generate a spike) when a strong perturbation arrives. Individual orbiting elements are oscillatory (in the reference frame fixed by the condensate) and perform spontaneous rotations.

Thus, the system can be understood to be composed of oscillatory elements and excitable elements with different thresholds: some active elements are in a suprathreshold state, repeatedly generating spikes, whereas some elements are in a subthreshold regime, susceptible to excitations. Threshold values for each element depend not only on the local topology of the network, but also on the phase shifts in the interactions of this element with its network neighbors.

Spontaneous spiking is periodic for $\mu_{2}<\mu<\mu_{1}$, as evidenced by the fact that the maximal Lyapunov exponent is zero $\left(\lambda_{1}=0\right)$ inside this interval. Actually, as seen in Fig. 3(b), several Lyapunov exponents can be zero inside this interval, indicating that many oscillators are orbiting independently. For the lower feedback intensities, the collective dynamics of the system becomes chaotic.

FIG. 6. (Color online) Instantaneous velocities of several randomly chosen elements $\mu=0.87$. 


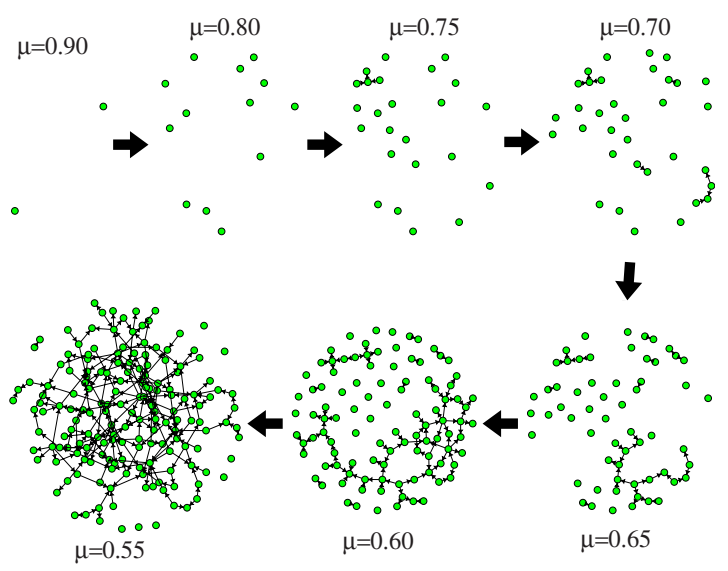

FIG. 7. (Color online) The sequence of active subnetworks observed under gradual decrease of the feedback intensity $\mu$.

\section{ACTIVE SUBNETWORKS}

When velocity synchronization breaks down and nonentrained active elements first appear, only a few of them are present. The number of active elements should however increase as the feedback intensity is further decreased. Some of them would have other active elements as their neighbors and, thus, active subnetworks would be formed. In this section we identify such subnetworks in the system and investigate how their properties depend on the feedback intensity.

To construct active subnetworks, we fix the global feedback intensity and let the system evolve. Analyzing its dynamics after a transient, the set of elements which become active is determined. The active elements occupy certain network nodes. Next we retain only these nodes and network connections between them, ignoring elements belonging to the synchronous condensate and their connections. In this way, the subnetworks of active elements are obtained.

Figure 7 shows the active subnetwork for several intensities $\mu$ of the global feedback. We see that initially the active subnetworks consist of a small number of isolated elements $(\mu=0.9$ and $\mu=0.8)$. As new active elements emerge at low feedback intensities, small network fragments with two, three, and four elements are formed $(\mu=0.75$ and 0.7$)$. The

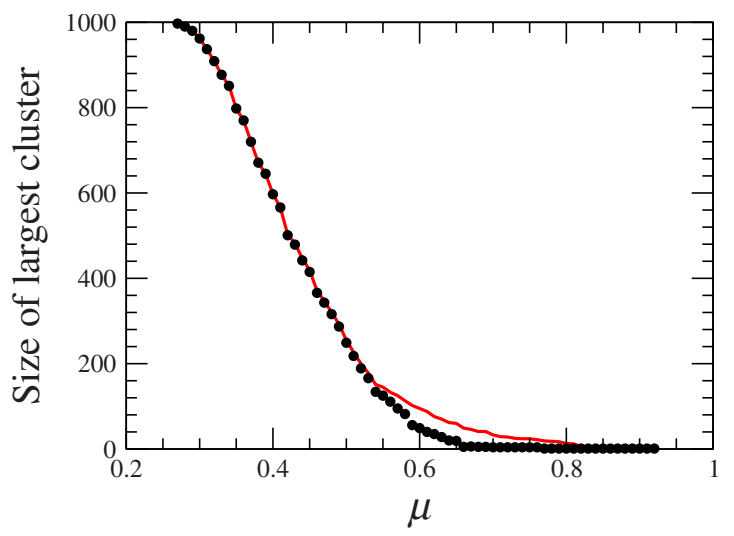

FIG. 8. (Color online) Dependence of the number of active elements (line) and the size of the largest connected active subnetwork (dots) on the feedback intensity $\mu$.

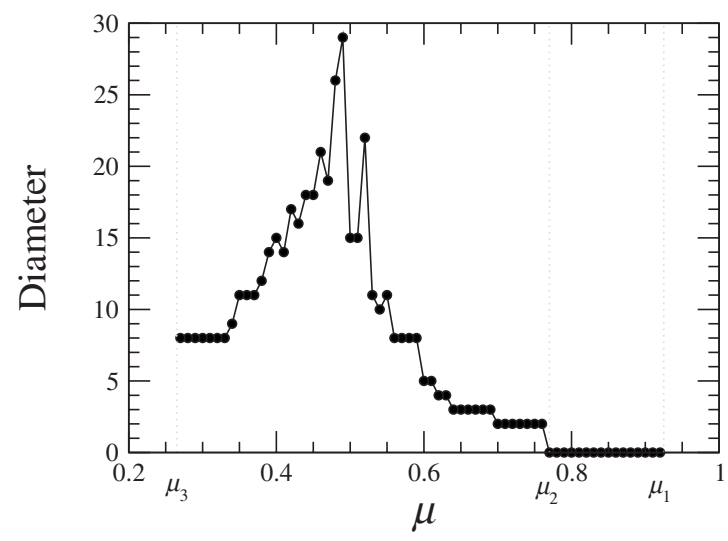

FIG. 9. The largest diameter (dots) of active subnetworks as function of the feedback intensity $\mu$.

connected network components increase in size $(\mu=0.65$ and $0.6)$ and finally a large connected component develops $(\mu$ $=0.55)$.

In Fig. 8, the total number of active elements and the size of the largest connected component are shown depending on the control parameter $\mu .^{2}$ Within a range of the control parameter, the size of the largest connected component is well below the number of active elements, implying that there are many disconnected network fragments present. For $\mu<0.53$, both curves become almost coincident, indicating that most of the active elements belong to a single giant connected component. A subnetwork is viewed as a connected component if any of its elements is linked to at least one other element in the subnetwork, neglecting interaction directions. ${ }^{3}$

Decreasing the intensity of the global feedback increases the size and complexity of subnetworks of active elements. Recalling the curve for the maximal Lyapunov exponent in Fig. 1, we notice that it becomes positive and chaos emerges already for $\mu<0.77$, before active subnetworks merge into a giant component. Thus, the dynamics of relatively small subnetworks can already be chaotic.

An important structural property of any network is its diameter, defined as the longest of the shortest paths connecting pairs of elements. For networks with directed connections, each link in a path should be traversed along its prescribed direction. If there are several disconnected fragments, their diameters can be independently computed. In Fig. 9, the longest diameter is displayed as a function of the global feedback intensity. The diameters of active subnetworks can be compared with the the diameter of the full network, which is equal to 8 in the case considered.

\footnotetext{
${ }^{2}$ Since the distinction between active elements and element conforming a synchronous condensate requires the clear existence of the latter, the classification might not be well defined when the majority of the elements are active. However, we have seen in Fig. 5 that a pronounced peak for the velocity of the condensate persists even when more than half of the elements have become active. Therefore, the values presented in Fig. 8 are valid for the very large portion of the range.

${ }^{3}$ Note that this condition is weaker than the one used for matrix $T$.
} 


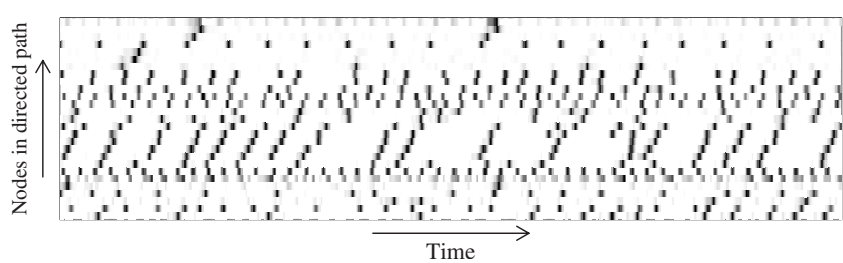

FIG. 10. Space-time diagram showing the dynamical activity pattern in the chain of 29 elements belonging to the active subnetwork.

As the feedback intensity is decreased, the largest diameter grows, starting from zero when only individual nodes are active. It reaches a maximum at $\mu \approx 0.49$ and then decreases. It is remarkable that, at its peak, the largest diameter of subnetworks is equal to 29 , which is much higher than that of the underlying network structure. After passing the maximum, the diameter takes the values close to 8 , the same as in the underlying structure.

Generally, active subnetworks are characterized by the presence of long paths and their diameters are significantly larger than those of the comparable random networks. For example, the subnetwork of diameter 29 has 293 nodes and its mean degree is 3.37. Standard directed random networks of this size and the same mean degree would have the diameter of about 23.

Active subnetworks are dynamical structures. Their elements perform repeated phase rotations with respect to the main condensate. Some of these elements are oscillatory and play the role of pacemakes. Other elements in a subnetwork are effectively excitable and their phase rotations are triggered by perturbations arriving at them from their neighbors. Thus, wave propagation takes place inside active subnetworks.

This kind of wave progagation is illustrated in Fig. 10. Here, we have taken the feedback intensity $\mu=0.49$ yielding the subnetwork with the largest diameter of 29 and have chosen the chain of nodes in the path corresponding to this diameter. The raster plot in Fig. 10 shows the dynamical activity along this chain. The absolute value of the velocities of the elements in the chain with respect to the synchronous condensate are displayed in gray scale, with the dark color corresponding to higher values. Repeated wave propagation along chain fragments is apparent. It can be also noticed that the entire activity pattern is irregular.

The elements forming an active subnetwork are strongly heterogeneous in terms of their intrinsic dynamics. The heterogeneity is caused by the fact that each element receives signals through its connections from a different number of elements-some of them active and others in the condensate - and these connections are characterized by different phase shifts.

The dynamics of an active element is described by Eq. (11). Separating in this equation the terms which correspond to the interactions of the chosen element with the condensate from those representing interactions with other active elements in the subnetwork, we can write it as

$$
\dot{\psi}_{i}=-\Omega+\frac{1}{2 i}\left[\left(\widetilde{z}_{i}^{c}+\bar{z}_{i}^{a}+\mu R\right) e^{-i \psi_{i}}-\text { c.c. }\right],
$$

where $\widetilde{z}_{i}^{c}$ and $z_{i}^{a}$ are local signals received by the considered element from its neighbors belonging to the condensate and to the active subnetwork, respectively. Introducing $R_{i}^{c}$ and $\Psi_{i}^{c}$ as $r_{i}^{c} \exp \left(i \Psi_{i}^{c}\right)=\mu R+\widetilde{z}_{i}^{c}$ and taking into account the definition (12) of local signals, the evolution equation for elements in an active subnetwork is

$$
\dot{\psi}_{i}=-\Omega+r_{i}^{c} \sin \left(\Psi_{i}^{c}-\psi_{i}\right)+\frac{1}{\rho N} \sum_{j=1}^{N} \sigma_{j} T_{i j} \sin \left(\psi_{j}-\psi_{i}+2 \pi A_{i j}\right) .
$$

Here, vector $\sigma_{i}$ defines what elements belong to the considered active subnetwork, i.e., $\sigma_{j}=1$ if the element $i$ is in the subnetwork and $\sigma_{j}=0$ otherwise. The second term in Eq. (16) describes interaction with the condensate and global feedback in the system. When the fraction of active elements in a network is relatively small, the global feedback is dominated by condensate elements.

Therefore, the condensate acts as external forcing on active elements. However, the magnitude $r_{i}^{c}$ of such forcing is different for different active elements, which explains the heterogeneity of emerging active networks. Those elements $i$, which have $r_{i}^{c}<\Omega$, are oscillatory and play the role of pacemakers. Their intrinsic oscillation frequencies are different and determined by the difference $\Omega-r_{i}^{c}$. On the other hand, the elements with $r_{i}^{c}>\Omega$ are excitable and their excitation thresholds are $h_{i}=r_{i}^{c}-\Omega$. For excitable elements in an active subnetwork, excitation thresholds are small enough, so that the excitation coming either directly from pacemakers or from the neighboring excitable elements is sufficient to trigger their phase rotation. For the element at the end of a wave propagation chain, all further network neighbors have thresholds so high that the excitation cannot be further transmitted.

Thus, inside an interval of feedback intensities preceding the final feedback breakdown, the considered system can generate a variety of dynamical networks whose sizes and dynamical properties can be controlled by the feedback intensity. These networks are effectively built from oscillatory and excitable elements. They are strongly heterogenous in terms of the oscillation frequencies and excitation thresholds of their elements.

As the feedback intensity $\mu$ is decreased, the number of active, nonentrained elements grows and they begin to dominate the dynamics of the system. In the next section we consider the behavior corresponding to the final loss of coherence in the transition.

\section{BREAKDOWN OF GLOBAL FEEDBACK}

Calculations of the maximal Lyapunov exponent and the embedding dimensions (Figs. 1 and 2) have indicated the presence of a singularity at about $\mu_{3}=0.26$. Below this point, both properties cease to depend on the feedback strength, thus suggesting feedback breakdown. Our investigations in Sec. IV have shown (Fig. 4) that this breakdown is also accompanied by a significant change in the distribution of 


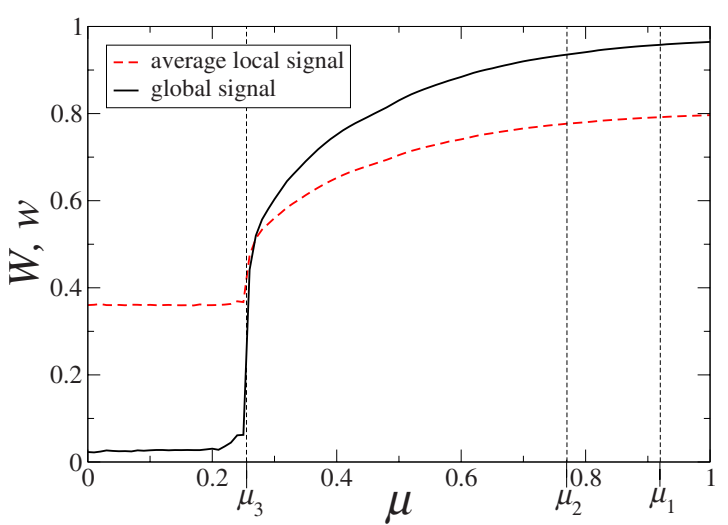

FIG. 11. (Color online) Time-averaged magnitude of the global signal (solid line) and mean time-averaged magnitude of local signals (dash line) as functions of the feedback intensity $\mu$.

average velocities. As the critical point $\mu=\mu_{3}$ is approached, the mean time-averaged velocity of the network sharply decreases. The dispersion of time-averaged velocities, i.e., the difference between the largest and the smallest average velocities observed, becomes large in the region preceding the transition. After the transition, both the mean velocity and its dispersion remain small and do not depend on $\mu$. Below in this section we provide further analysis of the feedback breakdown transition and dynamical properties of the network in its vicinity.

In Fig. 11, the computed dependence of the time-averaged global signal intensity $W=\langle|Z(t)|\rangle$ is shown. We see that it drops down to (almost) zero at the critical point and remains small below it. This provides direct evidence of the feedback breakdown. The global signal $Z=(1 / N) \sum_{i=1}^{N} \exp \left(\imath \phi_{i}\right)$ coincides with the Kuramoto order parameter of the considered oscillator population. Its vanishing indicates therefore the loss of synchronization at $\mu=\mu_{3}$. However, the observed phenomenon is essentially different from the Kuramoto desynchronization transition in globally coupled systems [5].

In oscillator populations with global, all-to-all coupling, vanishing of the Kuramoto order parameter leads to the loss of interactions between elements. They become almost independent, with the rest interactions inversely proportional to the size $N$ of the population and vanishing in the limit $N \rightarrow \infty$. This is not, however, the case in the considered network system.

The dashed line in Fig. 11 shows the computed dependence of the mean time-averaged local signal intensity $w$ on the feedback strength $\mu$, defined as

$$
w=\frac{1}{N} \sum_{i=1}^{N}\left\langle\left|z_{i}(t)\right|\right\rangle,
$$

where $z_{i}(t)$ is given by Eq. (10). We see that, while the mean local signal decreases together with the global signal as the critical point $\mu=\mu_{3}$ is approached, it does not vanish at this point. Below $\mu=\mu_{3}$, the mean local signal remains strong and does not depend on the feedback intensity.

Thus, local interactions do not disappear after the feedback breakdown transition in the considered network system.

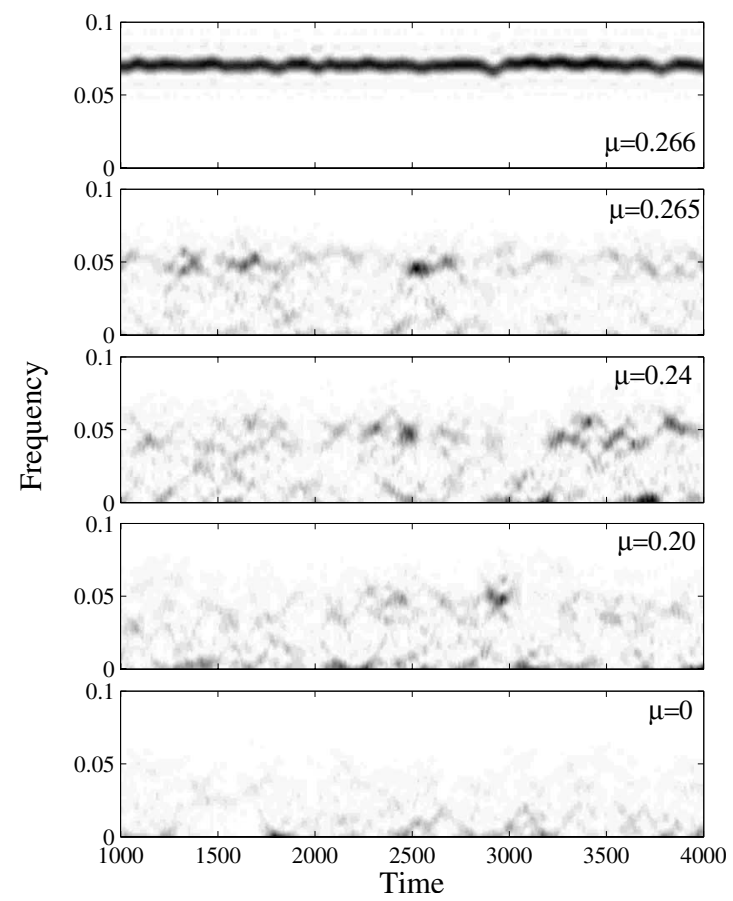

FIG. 12. Time-dependent spectral densities of the global signal at different feedback intensities $\mu$. The absolute magnitude of the signal is much larger at $\mu=0.266$, than in other plots shown.

Instead of full desynchronization-characteristic for globally coupled populations - the feedback breakdown leads the system to the highly dimensional chaotic state of network turbulence. Previously, analogous global feedback transitions have been investigated by Kawamura and Kuramoto [24] for continuous active media described by the complex GinzburgLandau equation. It is remarkable that, as seen in Figs. 1 and 2 , the maximal Lyapunov exponent and the embedding dimension increase as the critical point $\mu=\mu_{3}$ is approached and abruptly drop below it.

To investigate this transition, we characterize the system in terms of time-dependent power spectra, broadly employed in the statistical analysis of the brain EEG data (see, e.g., Ref. [25]). First, the autocorrelation function $S(\tau, t)$ of the global signal is calculated within a sliding time window of width $T$,

$$
S(\tau, t)=\frac{1}{T} \int_{t}^{t+T} Z\left(t^{\prime}\right) Z^{*}\left(t^{\prime}+\tau\right) d t^{\prime},
$$

After that, the time-dependent spectral density $S(\omega, t)$ of the global signal is determined by the Fourier transform

$$
S(\omega, t)=\left|\frac{1}{T} \int_{0}^{T} S(\tau, t) e^{-i \omega \tau} d \tau\right| .
$$

In our investigations we use a sliding time window of width $T=110$. In the plots showing the temporal evolution of the spectral density, a gray scale representation with the dark regions corresponding to the higher density is employed.

Figure 12 shows time dependent spectral densities for several selected values of the feedback intensity, both above and below the feedback breakdown. Above the transition 


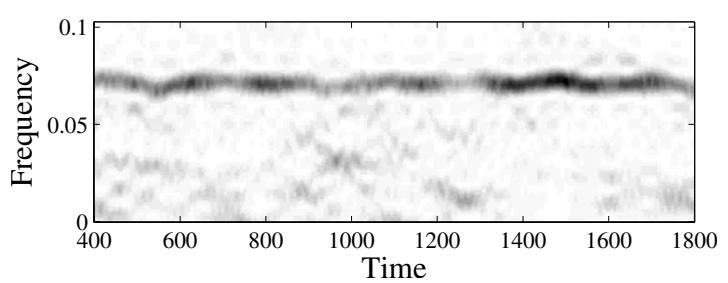

FIG. 13. Time-dependent spectral density of a single oscillator above the feedback breakdown transition $(\mu=0.266)$.

( $\mu=0.266$ ), a peak is persistently present in the spectral density of the global signal. The position of this peak corresponds to the frequency of coherent collective oscillations. At the critical point $(\mu=0.265)$, such persistent peak is absent. Instead, the system still exhibits irregular synchronization bursts seen as temporary maxima in the spectral density at the frequency of collective oscillations. This intermittent behavior is retained below the transition $(\mu=0.24,0.2)$. However, as the feedback intensity is gradually decreased, synchronization bursts become more rare and the characteristic duration of a burst decreases. Finally, in absence of the feedback $(\mu=0)$, spontaneous synchronization bursts have not been found.

It is interesting to compare spectral properties of the global signal with the respective dynamical properties of a single oscillator. The time-dependent power spectrum of an oscillator $i$ is defined as

$$
s_{i}(\omega, t)=\left|\frac{1}{T} \int_{0}^{T} s_{i}(\tau, t) e^{-i \omega \tau} d \tau\right|,
$$

where

$$
s_{i}(\tau, t)=\frac{1}{T} \int_{t}^{t+T} e^{i\left[\phi_{i}\left(t^{\prime}\right)-\phi_{i}\left(t^{\prime}+\tau\right)\right]} d t^{\prime} .
$$

Figure 13 shows the typical time-dependent power spectrum of an arbitrarily chosen element above the breakdown transition $(\mu=0.265)$. The spectral peak, observed in the spectral density of the global signal, is also visible in the spectrum of a single oscillator above the transition. This should indeed be expected if we take into account that all oscillators effectively experience external forcing generated by the global feedback, and their spectra therefore reflect the presence of such forcing.

Below the transition, the global signal becomes weak and does not exhibit strong influence on a typical individual oscillator. A detailed examination reveals however that some oscillators still (temporarily) possess a small maximum in their spectral density at the level of collective oscillations. Such oscillator groups may generate synchronization bursts visible in the spectral density of the global signal.

To test the conjecture that only some oscillator groups are responsible for synchronization bursts in the intermittent regime and that these groups are different in each next burst, a special investigation has been performed. We have chosen two different bursts at time positions and and calculated spectral densities $s_{i}\left(\omega, t_{1}\right)$ and $s_{i}\left(\omega, t_{2}\right)$ of all network elements individually. Then, we have compared the computed
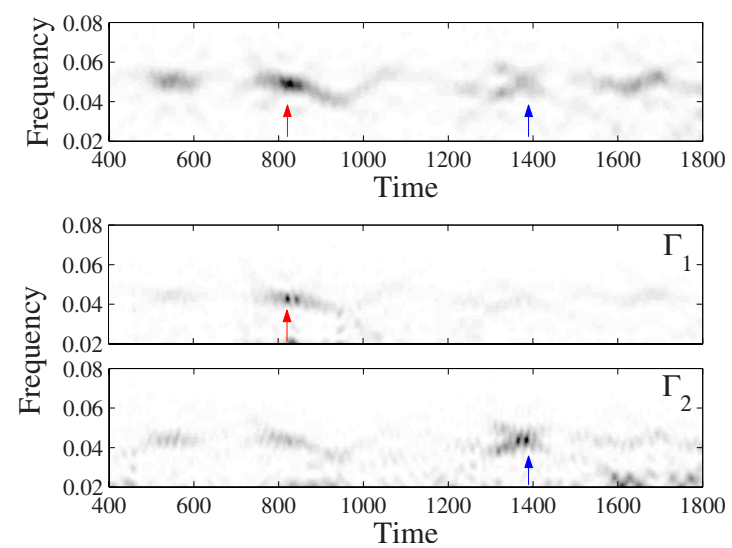

FIG. 14. (Color online) Time-dependent spectrum plot of (above) the global signal and (below) collective signals of two specially selected groups of oscillators. $\mu=0.265$.

spectral densities and selected groups of 50 oscillators at two times, whose spectra were closest to that of the global signal. To quantify the similarity between two spectral densities, we use the overlap

$$
q_{i}(t)=\frac{\int s_{i}(\omega, t) S(\omega, t) d \omega}{\int s_{i}(\omega, t) d \omega \int S(\omega, t) d \omega} .
$$

After each oscillator group $\Gamma$ of 50 elements has been selected, we have generated its summary signal

$$
Z_{\Gamma}(t)=\frac{1}{50} \sum_{i \in \Gamma} e^{i \phi_{i}(t)}
$$

and determined its time-dependent spectral density $S_{\Gamma}(\omega, t)$.

Figure 14 shows time-dependent spectral densities of the summary signals generated by two groups $\Gamma_{1}$ and $\Gamma_{2}$, which have been chosen as those mostly contributing to the spectral density of the global signal at $t_{1}=822$ and $t_{2}=1384$, respectively. For comparison, we also show in this figure the spectral density of the global signal within the considered time interval. Examining the figure, we see that, although traces of the spectrum of the global signal are visible in both partial summary spectra, the group of elements with the closest individual similarity to the global signal at a certain burst is already well collectively reproducing the global signal in the respective time interval. Thus, it seems plausible that only a subset of all network elements is strongly involved in the generation of a synchronization burst. Note that in the above analysis we have not attempted to identify all the elements in the respective groups and only demonstrated choosing a subset of 50 nearest elements is sufficient.

Analyzing composition of the two groups, corresponding to synchronization bursts at $t_{1}=822$ and $t_{2}=1384$, we find that they have only two common elements. Thus, the subset of partially synchronized elements is indeed different in different bursts in the intermittent turbulence regime. The elements of a group represent nodes in the considered network and there are links connecting them. Essentially, each group defines a certain subnetwork which temporally undergoes partial synchronization. Therefore, chaotic intermittency in the considered system can be understood as generating an 
irregular sequence of synchronization events imposing partial synchronization on different subnetworks. A synchronized subnetwork emerges, persists for some time, dissolves and, after a pause, is replaced by a different synchronized subnetwork.

\section{DISCUSSION AND CONCLUSIONS}

We have chosen a network model of coupled phase oscillators which exhibits highly dimensional chaos (network turbulence) and have shown how this chaos can be controlled and synchronization induced by introducing a global feedback. Our attention has been focused on the properties of dynamical patterns and intermittent turbulence characteristic for the transition region. An important property of the considered model was that individual elements were not chaotic and chaos emerged as a result of coupling between them.

Previously, investigations on global-feedback control of turbulence have been performed for continuous oscillatory media, in the general model of the complex GinzburgLandau equation $[9,10]$ and in experimental and theoretical studies of the catalytic CO oxidation reaction $[11,12]$. Action of global feedbacks on chaotic oscillatory systems with nonlocal coupling between elements has also been considered [26]. In all these systems, the individual oscillators were not chaotic and highly dimensional chaos (chemical turbulence) emerged as a result of diffusive coupling between them. These investigations have revealed that the behavior of oscillatory reaction-diffusion media on the edge of chaos is characterized by spontaneous appearance of various coherent activity patterns (see also the review [13]).

Specifically, propagating phase defects (kinks) spontaneously develop as the feedback intensity is decreased and cascades of reproducing kinks are observed. In a traveling kink, the phase undergoes a full $2 \pi$ rotation, or a slip $[9,13]$. Repeatedly appearing kink cascades destroy synchronous oscillations in the system and establish the state of intermittent turbulence.

Our work shows that in networks, the role of emerging coherent structures is played by active subnetworks. Some of the elements, forming such networks, spontaneously undergo repeated phase slips. Other elements produce phase slips in response to the excitation coming from their network neighbors. As result, traveling waves of phase slips repeatedly develop and propagate over a subnetwork, spreading from several emergent pacemaker centers. These traveling waves correspond to the cascades of kinks in continuous media.

While each next cascade in the continuous case is typically originating at a different spatial location and involves a different set of the elements of a medium, active networks are permanent in the considered network model. Although the patterns of wave activity in a subnetwork may be quite complex and chaotic, the subnetwork itself remains fixed.

This difference is due to the fact that the considered oscillator networks are strongly heterogeneous, with the heterogeneity imposed both by the structure of the network of connections and the phase shifts in interactions between individual elements. As a result of such heterogeneity, some elements become easily excitable and others turn into pace- makers. Together, such connected elements form permanent active subnetworks.

When wave patterns in active subnetworks are observed, the resting elements, representing the majority of a network, are in a synchronous state and form the condensate. As the feedback intensity is decreased, the number of active subnetworks increases and each network typically grows in size. In a percolation transition, individual subnetworks merge to form a giant active component, already comprising a substantial fraction of all network elements.

Starting from this point, distinguishing between active elements and the condensate becomes difficult. The system shows developed chaos and its embedding dimension is comparable to the total network size. Nonetheless, some intrinsic coherence is still present in its dynamics, as revealed by the persistence of the relatively strong Kuramoto order parameter characterizing synchronization.

As the feedback intensity is further decreased and approaches the critical point, the Kuramoto order parameter (almost) vanishes, indicating the loss of persistent synchronization. Since this order parameter yields at the same time the magnitude of the global signal, we conclude that breakdown of the feedback control takes place. Analogous feedback-breakdown transitions have previously been studied for continuous oscillatory media under the global feedback control [24]. In contrast to the global signal, local signals corresponding to network interactions do not vanish after the transition. Therefore, it is different from the desynchronization transition in globally coupled systems where, after the transition, interactions between elements almost vanish and the oscillators become effectively independent.

The time-dependent spectral analysis of the global signal has shown that, after its breakdown, the feedback is able to induce sporadic bursts of synchronization in the system. This behavior is reminiscent of what has previously been seen in the oscillatory systems comprising global and nonlocal coupling [26]. Our analysis suggest that different groups of elements are responsible for each next synchronization burst. Taking into account their connections, this means that different subnetworks of the entire network spontaneously exhibit (partial) synchronization. Each synchronization episode involves a different subnetwork and such episodes are alternating with complex asynchronous states.

This looks similar to the behavior characteristic for developed hydrodynamic turbulence, where different coherent structures are built and replace one another in an irregular sequence. In networks, spatial ordering is absent and therefore spatiotemporal patterns cannot obviously develop. Instead, coherent structures represent various dynamical subnetworks which get accentuated. The simplest form of coherence is partial synchronization, but other, more complicated kinds of coherent dynamics in the emerging networks are also possible.

The emphasis in this study has been on control of highdimensional network chaos, rather than on detailed investigations of network systems showing such kind of turbulence. We wanted to demonstrate that, by applying global feedback and adjusting its intensity, one can readily control the network dynamics. Therefore, we have chosen a particular model network, fixed its properties and then varied only the 
feedback parameters in our investigations. We have, however, checked that analogous results hold as well for other network realizations with various sizes.

While our investigations have been performed for a particular model, we conjecture that their results should be general and similar behavior will be found in other networkorganized systems of various origins. Indeed, it can be shown [27] that the considered model is closely related to the generic phase network model obtained by phase reduction of amplitude oscillator dynamics on networks in the vicinity of the supercritical Hopf bifurcation. The instability leading to the network turbulence in the model is the analog of the Benjamin-Feir instability of uniform oscillations in the complex Ginzburg-Landau equation.

\section{ACKNOWLEDGMENTS}

Stimulating discussions with Y. Kuramoto are gratefully acknowledged. Finacial support from the Volkswagen Foundation (Germany) is acknowleged.
[1] R. Albert and A.-L. Barabási, Rev. Mod. Phys. 74, 47 (2002).

[2] S. Boccaletti, V. Latoza, Y. Moreno, M. Chavez, and D.-U. Hwang, Phys. Rep. 424, 175 (2006).

[3] A. Arenas, A. Diaz-Guilera, J. Kurths, Y. Moreno, and C. Zhou, Phys. Rep. 496, 93 (2008).

[4] Chemical Waves and Patterns, edited by R. Kapral and K. Showalter (Kluwer, Dordrecht, 1995).

[5] Y. Kuramoto, Chemical Oscillations, Waves, and Turbulence (Springer, Berlin, 1984).

[6] A. S. Mikhailov, Foundations of Synergetics I. Distributed Active Systems, 2nd ed. (Springer, Berlin, 1994).

[7] A. S. Mikhailov and A. Yu. Loskutov, Foundations of Synergetics II. Chaos and Noise, 2nd ed. (Springer, Berlin, 1996).

[8] K. Kaneko and I. Tsuda, Complex Systems: Chaos and Beyond (Springer, Berlin, 2000).

[9] D. Battogtokh and A. Mikhailov, Physica D 90, 84 (1996).

[10] D. Battogtokh, A. Preusser, and A. Mikhailov, Physica D 106, 327 (1997).

[11] M. Kim, M. Bertram, M. Pollmann, A. von Oertzen, A. S. Mikhailov, H. H. Rotermund, and G. Ertl, Science 292, 1357 (2001).

[12] M. Bertram and A. S. Mikhailov, Phys. Rev. E 63, 066102
(2001).

[13] A. S. Mikhailov and K. Showalter, Phys. Rep. 425, 79 (2006).

[14] H. Daido, Phys. Rev. Lett. 68, 1073 (1992).

[15] E. M. Izhikevich, Phys. Rev. E 58, 905 (1998).

[16] P. Grassberger and I. Procaccia, Physica D 9, 189 (1983).

[17] G. Benettin, L. Galgani, A. Giorgilli, and J.-M. Strelcyn, Meccanica 21, 9 (1980).

[18] I. Shimada and T. Nagashima, Prog. Theor. Phys. 61, 1605 (1979).

[19] A. Wolf et al., Physica D 16, 285 (1985).

[20] E. Ott, Chaos in Dynamical Systems (Cambridge University Press, Cambridge, 1993).

[21] P. Frederickson, J. Kaplan, E. Yorke, and J. York, J. Differ. Equations 49, 185 (1983).

[22] B. Ermentrout, Neural Comput. 8, 979 (1996).

[23] G. Mato and I. Samengo, Neural Comput. 20, 2418 (2008).

[24] Y. Kawamura and Y. Kuramoto, Phys. Rev. E 69, 016202 (2004).

[25] E. Rodríguez et al., Nature (London) 397, 430 (1999).

[26] V. Casagrande and A. S. Mikhailov, Physica D 205, 154 (2005).

[27] H. Nakao and A. S. Mikhailov, e-print arXiv:092.3742v1. 